subsequently he took $\mathbf{8} \mathbf{g r}$. of theobromine twice daily. Whilst taking the diuretics the patient passed from 60 to $80 \mathrm{oz}$. of urine in the twenty-four hours ; previnusly it was below $500 z$. The oedeme visibly diminished, and the condition of the patient improved.

1 Comp. Rend. Soc. Biol, roja Rarerences.

d Comp. Rena. Soc. Biol, roju. 2 salknwski, Festschrift, rgo4. 3 Therap. 6 Comut Rend. Sic Biol f. Verdauungskrankh. 1931 9 Zeitschr. Int de Physiol, vol. i. 1904. '8 Arch. Soc. Biol., rgor. 11 Milnch. med. Woch., rg05. 11 Fortschr. d. Med., r897. 18 Loo. cit. 14 Hespitalstidende. 2904. 15 Zentralbl. f. inn. Med, 1005 16 Schweitz. Wooh. f Chem. u. Pharm.. 1897. 17 Die Therapie d. Gegenwart, 1902. 18 Berl. Klin. Woch., 1903. 19 Purin Bodies of Food Stuffs, rgo3.

\section{ON THE TREATMENT OF PURULENT CATITIES.*}

BY ROBERT H. WOODS, M.B., F.R.O.S.I.,

Burgeon for Diseases of the Throat, Nose, and Far to the Richmond Hospital, Dublin.

To judge by recent and current medical literature some confusion of thought exists concerning the principles underlying the treatment of purulent collections. One result of this confusion is that methods of treatment found suitable in particular cases are presumed to be applicable universally. Thus in every textbook I have seen, whether on general or departmental surgery, it is considered that the principle of drainage, so successful in dealing with soft-tissue abscesses, is a prime factor in the cure of chronic purulent collections inside fixed walls.

I shall attempt, among other things, to show that vimple drainage is of sinall value in such cases.

In every soft-tissue abscess the tissues which surround the abscess have been by its growth distorted from their natural position, to which place they always tend to revert. The force of this reversion, or, what is the same thing, the pressure of the pus, is determined as to its degree by the following three factors: First, the rapidity of the growth of the abscess; secondly, its situation; and, thirdly, its size.

As regards the first, a rapidly-growing collection pushes the tissues more rudely aside, it gives them less time to adapt themselves to its presence ; their natural elasticity is therefore less impaired than in a case of a slowly-growing one.

Secondly, the situation in which the abscess grows. If the surrounding tissues are soft and lax, the tendency to revert will be feebler and the pus pressure correspontingly lower than when the tissues are denser and tougher. By surrounding tissues is not necessarily meant only those directly bordering on the abscess, but all structures which tend to be distorted by its presence. Fibrous-tissue planes, though remote from the wall, may help it enormously in its reversion.

When we come to consider the influence of the mere size of the abscess on its pressure, we find the question is not quite - ) simple as it might first appear. It might be thought, since the bigger an abscess grows the more its surroundings are distorted, that the pressure inside would in all cases increase proportionately, but there is an important factor which interferes with this. In cases where we may regard the force of reversion, or pus pressure, as being due to tension in the sac wall, the pressure varies, according to a well-known physical law, not only as the tension per unit of length but also inversely as the radius of curvature.

$$
\mathbf{P}=\frac{\mathbf{T}}{\mathbf{R}} \dagger
$$

Therefore if the sac wall tension remains the same, the larger the sae, the lower will be the pressure inside and conversely. Now in a growing abscess the tissue fibres at the sac wall are always, except at the start, on the point of giving way and are in other words at their maximum tension Therefore I think we may say that the tension per unit of length of the sac wall is constant or nearly 80, for a given abscess, no matter what its size so long as it is confined to the same tissue.

This law of the pressure varying inversely as the radius only applies to cases where the pressure may be regarded as being due to tension in the sac wall, and where that tension is constant. In the other class-namely, where the pus pressure

\footnotetext{
- Read before the Surgical Section of the Royal Academy of Medicine, Ireland, on March ath

tFor the case of a cavity having a single curve. If the wall is curved in two directions the formula is $P=T\left(\frac{T}{R}+\frac{I}{R_{1}}\right)$ where $R$ and $R_{1}$ are the radii of currature.
}

is due to influences remote from the sac, it is by no means true.

If for simplicity's sake we suppose a small abscess situated in the middle of a much larger and not very resistant structure, which is in turn confined by a tough capsule, a relatively large increase in the abscess will only cause a relatively small stretching of the remote capsule, which will not be distorted beyond its limit of elasticity; its tension will be increased, and the pressure internal to it, and therefore the pus pressure increased. Hence we arrive at the interesting result that increase in the size of an abscess causes a fall of pressure if the resistance to its growth is immediately outside the sac, while if this resistance is some distance from the sac it results in a rise.

This is really only another way of saying that as long as the tissues, whether near or remote, to whose tension the abscess pressure is due, are not distorted beyond their limit of elasticity, increase in the size of an abscess is followed by a rise in its pressure, while if that limit be passed it results in a fall.

There is one possible case in which this is untrue, and though it does not occur in the human body, it may be mentioned for sake of completeness. The case would arise if the tissues were so elastic that the increase of tension due to stretching was more than counterbalanced by the increase in the radius of curvature.

It need hardly be said that though both these varieties exist in their pure form, yet most abscesses consist of a combination of both, in which one or another element predominates.

From the foregoing it only needs a little consideration to show that if the cavity is large enough and the surrounding tissues lax enough, or if the abscess is sufficiently. slow in its growth, the tendency. after incision to spontaneous evacuation of its contents may be so feeble as to be unable to overcome the effect of gravity if the opening is not near the lowest point; while if the abscess is moderate in size and the surrounding tissues firm, the influence of gravity, especially in acute cases, may in turn be quite negligible.

It is in the former class of case that drainage from, or close to, the most dependent point is so important ; for it is clear that the abscess will never heal if the head of the pus between the drainage opening and the lowest point of the sac is sufficient either to force the fluid further through the tissues, or to prevent the contractility of the tissues from extruding the matter. Examples of this are to be found in every part of the body-abscess beneath the scalp will occur to everyone. A chronic abscess burrowing through the thigh, especialiy when the wasting of the muscles from inaction has relaxed the deep fascia, is also a case in point; but perhaps the most common region is in and around the abdomen where the general looseness of the tissues favours its occurrence.

As an example of the latter class-that is, where the influence of gravity may be disregarded, a tonsillar abscess may be cited. The most convenient place for puncture is in the soft palate, and this is rarely the lowest point, yet the healing is never retarded on this account, for the abscess is generally acute, the quantity of pus is never very great, and the surrounding tissues are never very lax.

If the conditions are favourable even large abcesses may empty themselves in epite of the influence of gravity. I have seen two cases of deep-seated abscess at the root of the neck, which from the necessities of the cases had to be opened in front of the trachea low down, at a place which in the upright position must have been quite the highest point, and even in the recumbent position could not have been very far from it. From each of these a quantity of pus escaped on incision, which showed it must have come from deep within the chest, and yet in spite not only of the influence of gravity, but the perhaps more important negative pressure of the thorax, healing tosk place rapidly. In these cases the abcesses were situated in the connective tissue, and the extrusion of the contents was not so much the result of the contractility of the walls as of the reversion of the deep fascial places.

This negative pressure of the thorax leads us to consider the part it p'ays in the empyema of the pleural cavity.

The case of the pleura differs from any we have yet consided in that the outer concave wall is fixed or nearly so. Healing can only take place through obliteration of the cavity by union of the parietal and visceral walls. Now, this could only be accomplished rapidly by expansion of the lung in opposition to the negative pressure, a thing which the lung has no inducement whatever to do since the negative pressure of the thorax is in fact due to the contractility of the lung. So 
that for obliteration we have to depend on the growth of granulation tissue and the gradual falling in of the chest wall under the influence of the contraction of the fibrous layer deposited on its inner surface.

Here we have the exact converse of the conditions found in abscess beneath the scalp. Here, too, we have a fixed and a movable wall with a semilunar cavity between; but with this important difference, that it is the inner convex wall which is fixed and the outer wall which is movable. Here the coniraction of the movable wall tends to obliterate the cavity, while in the case of the pleura it has exactly the opposite effect. This is the sole reason of the difference of difficulty in getting the two cases to heal.

The negative pressure of the thorax is the constant factor in the enlargement of the pleural cavity by contraction of the lung, but in all except recent cases there is another. When the visceral pleura becomes thickened and the organized new tissue tends to contract, its effect is to aid the collapse of the lung, since its concavity is directed towards the root of the lung and away from the pleural cavity. This effect may be very small if the empyema is localized by adhesions, for the contracted surface will be only the chord of a very flat arc, but if the whole cavity be involved the effect will be very great.

From the point of view of its squeezing effect on the lung, this new contracting sheet is of importance. In the first place, the thicker it grows and the stronger it gets the harder it will compress the lung; but, in addition to this, its crushing power is increased by the mere fact that the lung get: gmaller, because decrease in the radius of curvature of $a$ contracting sheet means increase in the pressure to which the tension in that sheet gives rise. The delicate lung tissue can offer no resistance to the influence of this vicious circle, and we need not doubt that the process of carnification is entirely due to this cause. It is no wonder, then, if such a pleural cavity when opened takes an indefinite time to heal, for the effect of dependent drainage is purely negative.

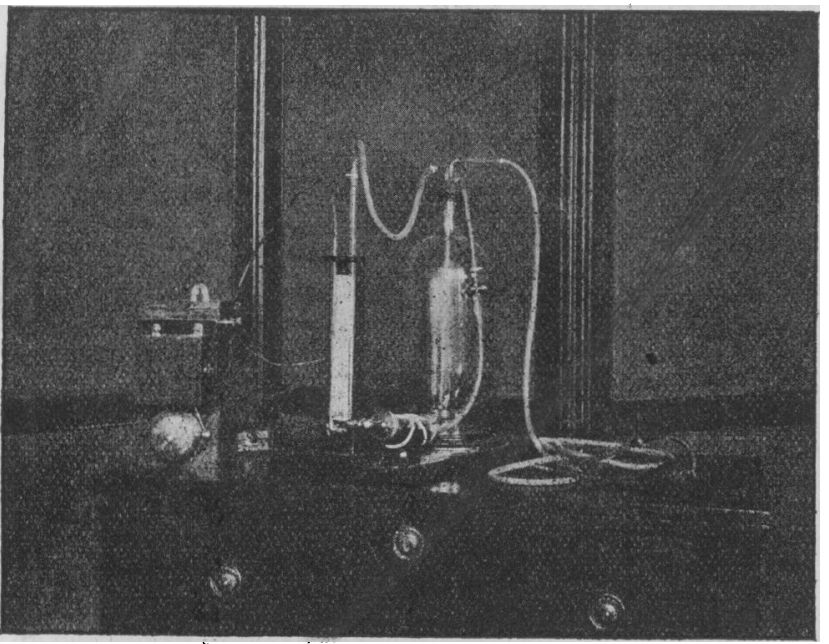

Up to the present the operation of resection of the ribs as advocated by Estländer is the most important surgical aid to the healing process. It helps the collapse of the rigid outer wall and so facilitates the obliteration of the cavity.

Let us now consider what would be necessary in order to make such a cavity heal at once. It is clear from the foregoing that if, while providing an exit for the pus, we could keep the lung continuously distended so that the parietal and visceral layers were in contact, fusion of the granulating surfaces would take place very rapidly, since there would be no cavity to be filled.

Now there are two ways of keeping the lung distended, and two only. The first is by corking up one bronchus and forcing air into the lung through a perforation in the cork. The other is by applying a suction apparatus to the pleural cavity; and I make this proposal believing it to be quite feasible, for even if some technical difficulties stand in the way, it is worth while to try and surmount them when we consider the importance of the end to be attained, namely, aborting the disease, and leaving the patient from the very first with a lung not more impaired than it already was by the disease which occasioned the empyema.
In the first place, the seat of the wound where the drainage tube is inserted should be made airtight. This could be managed by a small pneumatic pad applied round the wound. Through the middle of the pad the drainage tube should pass, the hole in the pad being hermetically sealed to the outer wall of the tube. Suction could be applied by an aspirated reservoir with a manometer tube attached.

In order to fix our ideas, as well as for the purpose of experiment, I have had such an apparatus made for me by Messrs. Smith and Sheppard. It consists of a large bottle, which can be sufficiently exhausted by a small air-pump. It is advisable that the capacity of the bottle should be considerable so that minute leakages, such as are inevitable with rubber tubes and joints, as wtll as the gradual outflow of pus to take the place of air, may have but a small influence on the pressure.

The pressure is gauged by means of a U-shaped mercurial manometer. Into the free end of the manometer a pair of wires, connected with an electric alarm, are lowes ed to any requisite depth. Should the negative pressure in the reservoir fall below a certain point corresponding with the tips of the wires, the mercury rises, makes contact between the points, and the attending nurse is summoned by the bell.

The air pad, which is fixed to the drainage tube, is secured to the patlent not only by a belt going round the chest, but locally by rubber strapping.

$I$ have had pads made in various shapes and sizes. The best form can only be decided experimentally, but the most promising is a conical one with the drainage tube passing through the axis of the cone. The pad need not necessarily contain air. Water would be less liable to leakage, besides avoiding the necessity of opening the pad to allow for expansion when sterilizing by boiling. Great care should be taken to allow no air to enter the pleural cavity either before or during treatment. If it entered it would rise to the highest part of the cavity and prevent the union of the surfaces between which it lay. Furthermore, it would stand no chance of being removed by an apparatus arranged only to abstract the heavy material from the most dependent part. If air gains admission it can be removed by turning the patient on the opposite side and filling the pleural sac with boric lotion, at the same time providing for the escape of air.

It is therefore evident that once the pad has been put in position and the treatment started it ought not to be removed until the case is quite or very nearly cured, for removal of the pad would mean entry of air and ripping up of the adhesions between the granulating surfaces. For the same reason the tube leading from the pad to the bottle should be carefully clamped before removing the bottle for emptying and cleansing, and the bottle again exhausted before undoing the clamp.

Theoretically, the opening in the chest wall should be made at the most dependent point as the patient lies, so that the gravitation of the pus might cause the adhesion of the pleural layers to begin at parts remote from the opening, which should, of course, be the last place to close. But practically, I think, it will be found sufficient if the opening is some distance away, provided the . nd of the empyems tube is at the bottom of the cavity. When the rest of the pleura has adhered, the track can be made to close by shortening the tube.

Again, the earlier in the history of such a case the treatment were tried the better would be the chance of success, for the more recent the visceral exudation the less it will be organized, and the less will be its tendency to contract. With regard to the degree of negative pressure to be applied, it must be at least sufficient to expand the lung. If the method were applied at the onset of the disease, the normal negative pressure of the thorax would be the only resistance to be overcome by the pump. But since, in many cases, the correct amount will not be precisely known, a greater negative pressure must, for certainty sake, be resorted to, and there is no reason. as far as one can see, against the minimum being considerably exceeded. The determination of the most useful pressure must, however, be left to experience and experiment.

An approximation to the correct degree may be made by watching the effect of gradual increase of negative pressure on the outflow of pus. When it is seen that a further increase is not responded to by an additional outflow, it may be taken as proof that the existing amount is enough to keep the cavity nearly empty.

In pyopericarditis, in the same way the negative pressure of the thorax keeps the parietal pericardium distended, and thus 
retards healieg by preventing its contact with the visceral layer.

When we come to rigid cavities, such as the antrum of Highmore, where the mucous membrane is degenerated, the tutility of expecting drainage to effect a cure will, from previous considerations, be apparent. It may be admitted that in the history of a case of empyema of the antrum from simple infection - that is, where there is no tooth or bone disease, there is a point up to which simple drainage might allow the lining membrane to recover, though I am not at all aware that this has ever been tried. But when once the parulent stage is established it is hard to see how this can ever occur. The truth is that mere drainage, if not actually parnicious in such cases, can be entirely dispensed with. At best it can only get rid of excess of pus; it can never leave the cavity pus-free.

Further, in cases where the membrane at the hiatus semilunaris is degenerated or swollen, no ordinary-sized hole would have the effect of emptying the antrum, for no air could come in from above to take the place of the pus. The rule that lays down that the antra should be tapped at the most dependent point is only an unreasoning extension of what in other circumstances is a highly important practice. The real reason why opening the antram from below sometimes cures empyema is that it allows thorough flashing and cleansing of the cavity, and by removing the infected matter permits the lining membrane to recover. This end is just as well attained with a solid plug in the opening as with a drainage tube, while the plug has the additional advantage that the pus does not trickle into the mouth and get swallowed by the patient. In cases where degeneration is so well established that regular and frequent flushing does not effect a cure within a reasonable time, continuing the treatment.will not bring it about.

Granulation tissue would be no bar to healing if the walls could but collapse and the granulations fuse, but the rigidity of the walls prevents anything of the kind from happening for after the first few layers granulations grow so slowly that it would take an enormous time for obliteration to be effected in such a way.

An effort must then be made to curette the diseased tissue and allow the raw surfaces to epithelialize. This can be effected by cutting away the inner wall of the antrum to such an extent as to permit the mucous membrane of the nose to creep in and cover the raw surfaces with a healthy coat. I have lately in three cases supplemented this method by applying large grafts to the inside of the antrum through the opening in the canine fossa a week after the primary operation, and in each case with an excellent result.

Again, let us take the case of the frontal sinus. Here we have the best possible example of - the. insufficiency of drainage to cure an infected bone cavity. The sinus is provided with an exit at its most dependent point, and if drainage were of any service whatever, one can hardly see how such a cavity could ever become chronically affected. The explanation given by those who attribute its disease to want of proper drainage is that the concomitant swelling of the mucous membrane of the infundibulum blocks the exit, and so. impedes the gravitation of the discharge. So in many cases it undoubtedly does, and in these we have brow ague from pus pressure as a constant symptom; but in many others the infundibulum is large enough to allow perfect drainage, in spite of swelling, and this is borne out by the ease with which large instruments can be introduced into the cavity from the nose, and also by the complete absence of frontal ache, a symptom which could not fail to be present were the outflow in any way obstructed.

That such cases do not tend to spontaneous cure shows that mere drainage is not enough, unless the other necessary condition is present-namely, that one wall of the cavity must be collapsible.

Here, then, we have the key to the correct treatment of such oases. There is no use in trephining the anterior wall and scraping and disinfecting the cavity. The mucous membrane has been destroyed, and when the bone granulates the old condition is re-established as badly as ever. In the same way a diseased mastoid process will never heal from simply letting the pus out by chiselling if its interior is degenerated. Such treatment should be reserved for acute cases, where, on the subsidence of the inflammation, the membrane reverts to its normal condition.

Operations on the mastoid in old-standing cases were heartbreaking to the surgeons until the modern radical procedure was developed. The essence of this beautiful operation consists in throwing the tympanum, external meatus, mastoid cells and attic, all into one large accessible cavity over the surface of which epidermis is encouraged to grow by utilizing the skin of the external meatus and by transplantation by Thiersch's method from other parts of the body.

So, also, when abscess occurs in bone, the head of the tibia for example, opening and scraping and drainage are of no use, the granulations cannot fuse, for they cannot meet if the cavity is of any but the smallest size. The efforts of the surgeon should be directed towards laying the cavity freely open and planting the raw surface with large grafts.

In the majority of surgical cases pain is a function of tension. Now, tension in cases of infection is determined by two things: the rigidity of the surrounding tissues and the time they get to adapt themselves to their new conditions. There are certain parts of the body noted for their painfulness when inflamed. These are not necessarily places richly supplied with sensory nerves, but rather parts whose structure is such that it does not readily allow of distortion.

A gouty or rheumatic inflammation of fibrous tissue is always severe, and a patient may suffer a degree of torture from a small furuncle on the fibro-cartilaginous parts of the nose or ear out of all proportion to the size of the affected area. If the affection is virulent the inflammatory products accumulate rapidly, the mechanical disturbance of the tissues is violent, the tension is high, and the pain great. If, on the other hand, the infection is mild the products accumulate slowly, the tissues get time to adapt themselves, the tension is low, and there is but little pain, and if the malady only progresses with sufficient slowness there is no reason why pain should not be entirely absent.

This gives a more reasonable explanation of the fact that taberculous otitis usually progresses painlessly, than the assumption, obviously made to fit the fact, that the tubercle bacillus produces a special anaesthetic toxin which deacens the nerves of the affected area.

\section{FRONTAL SINUS FMPYEMA EOLLOWED BY SUBDURAL ABSCESS.*}

Bæ. A. J; MARTINEAU, F.R.C.S.EDIN.,

Assistant Burgeon to the Throat and Ear Hospital, Brighton.

J. M., aged 31, was first seen in January, 1904, when he complained of pain in the right cheek, upper molar teeth, and over the right eye, and of a nasal discharge. Symptoms had been present five or six months. He had been to a dentist and had a sound tooth drawn.

On examination there was found a marked deviation of the nasal septum to the left side and a corresponding enlargement of the turbinar bones on the right. There was pus about the middle turbinal region and the cheek was dark on transillumination.

On January 29th, 1904, the right antrum was perforated through the tooth socket under gas and foul pus washed out. The patient was tanght to wash the cavity out himself, and this antrum gave no more trouble. In the following August the left antrum began to trouble him. Symptoms were the same as he had had on the right side, and I saw him on August 23rd. He had had a molar tooth extracted before. I saw him on this occasion also, and there was said to have been an abscess at its root. (I may mention that all his teeth are unusually good.) I perforated this antrum also through the tooth socket, and daily syringing was instituted.

I was called to see him one week later for acute pain on the left side of the forehead and round the orbit, especially at the inner angle of the lids. His temperature was $101^{\circ} \mathrm{F}$. The antrum when examined washed out clear. The day before he had been for a long motor-car ride, which, owing to a mishap, had been prolonged until 2 a.m. on the day when I saw him. Pain commenced at 6 a.m.

I tried to pass a frontal sinus cannula under cocaine and failed. I put him on inhalations of mentholized steam, and tried again, but without success.

On September Ist, under chloroform, I raised an angular flap over the sinus, and removed a circle of bone with a $\frac{1}{4}$-inch trephine. The mucous membrane lining the anterior wall was deeply congested, and gas bubbled through a puncture made in it by the pin of the trephine. The sinus was full of offensive yellow pus, and there was a small area of bare: bone on the inner wall. It was ascertained that a probe could be passed into the nose, and the inferior turbinal was removed *Case shown to the Brighton Divisiop of the British Medical Association. 